Open Access

\title{
Molecular cytogenetic studies characterizing a novel complex karyotype with an uncommon $5 q 22$ deletion in childhood acute myeloid leukemia
}

\author{
Amanda Faria de Figueiredo ${ }^{1,2}$, Roberto Rodrigues Capela de Matos ${ }^{1,2}$, Moneeb A. K. Othman ${ }^{3}$, Thomas Liehr ${ }^{3}$, \\ Elaine Sobral da Costa ${ }^{4,5}$, Marcelo Geradin Poirot Land ${ }^{4,5}$, Raul C. Ribeiro ${ }^{6}$ Eliana Abdelhay ${ }^{2,7}$ and Maria Luiza Macedo Silva $a^{1,2^{*}}$
}

\begin{abstract}
Deletions in the long arm of chromosome 5 or loss of the whole chromosome are rare in childhood Acute Myeloid Leukemia (AML) patients. It is also unknown if the wide variety of breakpoints have diverging implications in the patient's outcome. Despite $-5 / 5 q$ - abnormalities have usually been described as a poor prognostic feature, however, the low frequency of $-5 / 5 q$ - in pediatric AML patients limits a full knowledge about this cytogenetic and clinical category, which is an intriguing factor for further research and new findings. Here, we report an AML child showing an uncommon deletion in $5 q$ associated with 2 new abnormalities involving chromosome 2 within a complex karyotype well-characterized by several molecular cytogenetic approaches. Our work stimulates upcoming studies with more detailed descriptions about $5 q$ abnormalities to better define its role in the stratification risk of such cytogenetic subgroup in childhood AML.
\end{abstract}

Keywords: Childhood AML, Uncommon deletion 5q22, Complex karyotype

\section{Background}

Several studies in childhood acute myeloid leukemia (AML) have been showing three prognostic cytogenetic group classification: favorable cytogenetics, that includes $\mathrm{t}(8 ; 21), \mathrm{t}(15 ; 17)$ and inv(16), high-risk cytogenetics, that includes complex karyotypes, monosomy 7 , monosomy 5 , $\operatorname{del}(5)(\mathrm{q})$ or abnormalities in 3q, and intermediate risk: other changes [1]. The subgroup that has $-5 / 5 q$ rearrangements is rare and comprises about $1-2.5 \%$ of pediatric AML cases [2]. This finding was recently confirmed by Johnston et al., which described cytogenetics data of 26 pediatric patients $(1.2 \%)$ with $-5 / 5 q$ abnormalities in a retrospective study, including 2240 children and observed that this cytogenetic subgroup presented a very poor outcome [3]. Despite of a concise and comparative study in a

\footnotetext{
* Correspondence: mlmacedosilva@hotmail.com

'Bone Marrow Unit, Cytogenetics Department, National Cancer Institute, Rio de Janeiro, Brazil

${ }^{2}$ Post-Graduation Program in Oncology, National Cancer Institute, Rio de Janeiro, Brazil

Full list of author information is available at the end of the article
}

large cohort of childhood AML, the low frequency of 5qcases limit a full comprehension of the cytogenetic and clinical implications of this subgroup [1, 3, 4]. Thus, to contribute to the understanding of this rare subgroup within childhood AML, we describe the clinical, Gbanding and molecular cytogenetic data of a child with AML showing an uncommon deletion $5 \mathrm{q} 22$ in a new complex karyotype.

\section{Case presentation \\ Case report}

A 10-year-old boy was admitted with a 4-months history of fever, generalized lymphadenopathies and weight loss. Physical examination revealed mucositis and gingival hyperplasia. The abdomen was painless with no palpable mass. Imaging examination revealed mediastinal widening. At admission, the hemoglobin was $7.2 \mathrm{~g} / \mathrm{L}$, white blood cell count was $67.5 \times 10^{9} / \mathrm{L}$ and platelet count was $275 \times 10^{9} / \mathrm{L}$. The peripheral blood smear showed $80 \%$ of blast cells. Bone marrow was hypercellular with 94,6 \% FAB-M1 myeloid blast cells, being $93,7 \%$ of granulocytic 
progenitors $\left(\mathrm{CD}_{34} 4^{\text {hi }} ; \mathrm{MPO}^{-1+(50 \%)}\right.$; CD117 ${ }^{\text {hi }}$; HLA-DR ${ }^{\text {hi }}$; CD13 ${ }^{\text {hi }}$; CD36 ${ }^{-}$; CD11b ${ }^{-}$; CD16 ${ }^{-}$; $\mathrm{CD}_{4}^{-}$; CD35- ${ }^{-}$CD14 ${ }^{-}$; IREM-; CD71 ${ }^{\text {lo }}$; CD105 $; 5^{-} \mathrm{CD}^{-/+(50 \%)}$; $\mathrm{TdT}^{+} ; \mathrm{CD}^{-1+(5 \%)}$; $\mathrm{CD} 38^{+}, \mathrm{CD} 2^{+} ; \mathrm{CD} 15^{+}$). The patient was initially classified for intermediate-risk arm, and treated on AML-BFM 2012 protocol [5].

Morphological examination of the bone marrow on days 15 and 33 of induction chemotherapy revealed myeloid blast cells comprising $>25 \%$ (M3 bone marrow evaluation) and M2 ( $>5$ to $25 \%)$, respectively. At the 42nd day from the beginning of the treatment, the blast cell percentage in the bone marrow was $21,6 \%$. The patient achieved a late complete remission $(0.7 \%$ blast cells, < $5 \%$ - M1) only on day 79 of treatment. Due to the persistence of blast cells after the second induction (42nd day from the beginning of the treatment), the patient was stratified to high-risk arm, and was submitted to allogeneic stem cell transplantation, as recommend by the protocol [5]. This procedure was well tolerated and the patient is in continuous complete remission for eight months now and twelve months since initial diagnosis. This study was approved by the Ethics Committee of the Brazilian National Cancer Institute (CEP \#088/07).

\section{Methods and results}

G-banding studies revealed a karyotype 46,XY,der(2)? $t(2 ; 15)$, del(5), der(14)?add(14)(q23) in 15 of 20 metaphases analyzed (Figs. 1a, b and c). Several molecular approaches were performed to discover this complex karyotype. FISH was performed using the LSI EGR1 SpectrumOrange/D5S23 (5q31), D5S721 SpectrumGreen (5p15) probes (Vysis) and showed a heterozygous EGR1 deletion in 10 metaphases analyzed and interphase nuclei analysis revealed the same pattern in 150 of 200 analyzed cells (Fig. 1d). Although FISH using LSI IGH break apart showed no split signal (Fig. 1e), the position of IGH normal signal suggested that there was a translocation between the chromosomes 2 and 14 (Fig. 1e). Whole Chromosome Painting (WCP) probes for chromosomes 2, 14 and 15 were also applied and the results revealed a translocation between chromosomes 2 and 14 and also between the other homolog of chromosome 2 and chromosome 15, therefore the karyotype had no normal chromosome 2 (Fig. 1f). Further, Multicolor Chromosome Banding (MCB) was performed for chromosomes 2, 5, 14 and 15, as previously reported [6]. Overall, MCB characterized a complex chromosomal alteration between chromosomes 2, 14 and 15 (Fig. 1g). In order to define all the breakpoints involved in this complex translocation, mainly $5 q_{-}$, it was necessary to apply several Bacterial Artificial Chromosome (BAC) probes (Table 1). The final karyotype was characterized as: 46,XY,t(2;14)(q23.1;q32.2),t(2:15)(p22.3;q21.1), del(5)(q22qter). The karyotype was described according to the
International System for Human Cytogenetic Nomenclature [7].

\section{Discussion}

Abnormalities of chromosome 5 are a common finding in patients with hematological malignancies with poor outcome [8]. The deletion of the long arm of chromosome 5 and monosomy of 5 has been described most frequently in Myelodysplastic Syndrome (MDS) cases. However, it has been already documented as a recurring finding in AML and it is associated with dismal outcome [9]. 5q deletion has been shown a wide variety of breakpoints and it has been reported within complex karyotypes.

Johnston and coworkers described in a wide retrospective cohort of 2240 pediatric AML patients, that 5q deletion was also associated with poor outcome, in which to have more than three cytogenetic abnormalities showed worse outcomes than those with three or fewer cytogenetic abnormalities [3]. On the other hand, it is important to remark that $7 / 26$ patients, from the above-mentioned work were classified as harboring unbalanced rearrangements resulting in $5 \mathrm{q}$ loss, all of that without thoroughly refine the karyotype in the search for the presence of cryptic abnormalities, which may be particularly hard to detect in G-banding cytogenetics.

In the present work, the patient carries on the deletion of chromosome 5 within a complex karyotype and has lost a large portion from the long arm of one of the chromosomes 5. Initially, the FISH assay, with LSI probes, demonstrated the deletion of 5q31, but with FISH using several BAC probes (Table 1) and MCB approaches, we could detect the breakpoint in $5 \mathrm{q} 22$ which is an uncommon finding in patients harboring $5 \mathrm{q}$ deletion suggested by LSI FISH analysis.

Volkert et al., suggested that the type of cytogenetic abnormality leading to loss of $5 \mathrm{q}$ may harbor important prognostic information. A wide series of MSD and AML adult patients were taken into account in this work. This study showed that the two main commonly deleted regions (CDR) have distinct prognostic value. The CDR1 (5q32) is present in patients with $5 q-$ MDS and isolated $5 \mathrm{q}$ deletion, being associated with a good prognosis. The CDR2 (5q31) is present in aggressive MDS and high-risk $\mathrm{AML}$, and has been associated to complex karyotypes and demonstrated a worse prognosis [9].

Interestingly, our patient presented a loss of large portions from the long arm of one of the chromosome 5 that comprised 5q22 to 5qter, including both CDR regions. In this large portion lost in our patient, the literature includes APC, EGR1, CTNNA1, DIAPH1, NPM1, GLRA1, RPS14, UBE2D2 genes whose disruption of expression may lead to defects in hematopoiesis in mice and other defects in core process to cell development. Lastly, these 


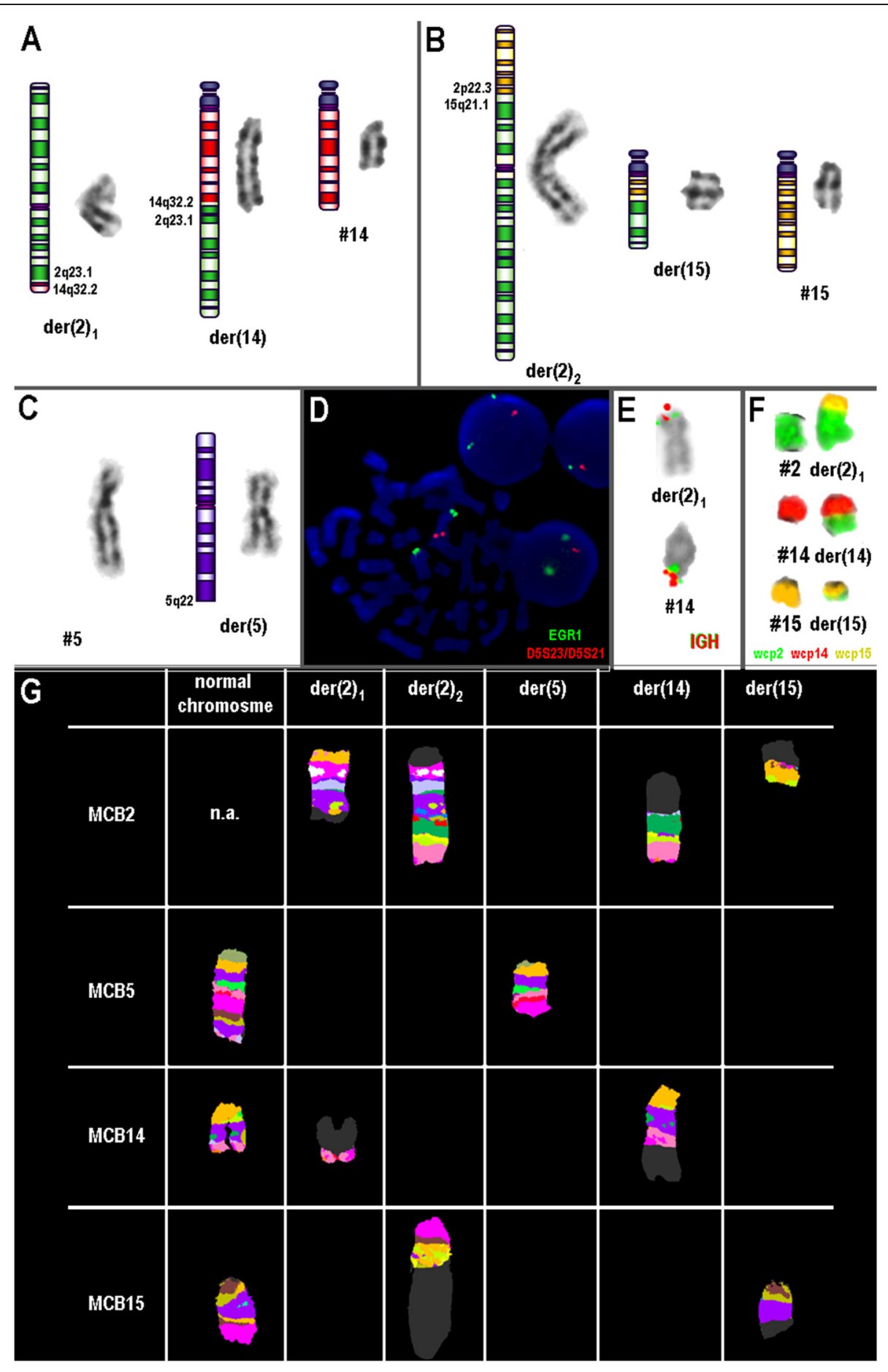

Fig. 1 a, b, and $\mathbf{c}$ show the final partial G-banding karyotype. a t(2;15)(p22.3;q21.1); b del(5)(q22); c t(2;14)(q23.1; 32.2$)$. d FISH with LSI 5p15/5q31 (EGR1) showing a deletion of the red signal (5q31) in a metaphase and in nuclei interphase. e FISH with WCP for chromosome 2 (green), 14 (red) and 15 (yellow) shows translocation between chromosomes 2, 14 and 15 (R-DAPI image). $\mathbf{f}$ FISH-results characterizing the normal and derivative chromosomes 2, 5, 14 and 15 are summarized. In the first line results of MCB for chromosome 2 are depicted and show both derivatives 2 and derivative 14 and 15. The second line shows the normal chromosome 5 and a derivative of chromosome 5. The third line shows the normal chromosome 14 and the derivative chromosomes 2 and 14 . The fourth line shows the normal chromosome 15 and the derivative chromosomes 2 and 15. Here, MCB probe sets for chromosomes 2, 5, 14 and 15 were used. According to FISH and MCB results, the karyotype was re-interpreted as 46,XY, t(2;14)(q23.1;q32.2), t(2:15)(p22.3;q21.1) and del(5)(q22-qter) 
Table 1 Probes and BACs applied in the case to characterize the breakpoint in each chromosome involved

\begin{tabular}{|c|c|c|c|}
\hline Cytoband & Position [hg19] & Probe & Results in derivative chromosomes \\
\hline $2 p 24.3$ & chr2: $16,014,784-16,140,647$ & RP11-119F22 & Signal on der(15); no split signal \\
\hline $2 q 23.3$ & chr2: $26,967,697-27,136,688$ & RP11-106G13 & Signal on der(15); no split signal \\
\hline $2 \mathrm{p} 23.2$ & chr2: $29,415,640-29,447,593$ & SPEC ALK & Signal on der(15); no split signal \\
\hline $2 p 22.3$ & chr2:35,864,069-36,032,088 & RP11-119B15 & Signal on both der(2); no split signal \\
\hline $2 q 22.3$ & chr2:145,181,324-145,355,222 & RP11-64O2 & Signal split on $\operatorname{der}(2)$ and on der(14) \\
\hline $2 q 23.3$ & chr2:153,589,449-153,743,069 & RP11-58K7 & Signal on der(14); no split signal \\
\hline $5 q 15$ & chr5:93,905,245-93,906,381 & RP11-461G12 & Signal on der(5) \\
\hline $5 q 15$ & chr5:95,549,050-95,550,779 & RP11-254I22 & Signal on der(5) \\
\hline $5 q 15$ & chr5:97,013,251-97,014,385 & RP11-72K17 & Signal on der(5) \\
\hline $5 q 21.1$ & chr5:98,701,067-98,853,238 & $\mathrm{RP} 11-102 \mathrm{H} 6$ & Signal on der(5) \\
\hline $5 q 21.1$ & chr5:100,241,673-100,434,269 & $\mathrm{RP} 11-109 \mathrm{H} 23$ & Signal on der(5) \\
\hline $5 q 21.3$ & chr5:106,587,839-106,772,024 & CTD-2337D22 & Signal on der(5) \\
\hline $5 q 22.2$ & chr5:112,045,171-112,208,641 & RP11-107C15 & Signal deleted on der(5) \\
\hline $5 q 22.2$ & chr5:112,460,936-112,667,499 & RP11-467F22 & Signal deleted on der(5) \\
\hline $5 q 22.3$ & chr5:114,413,269-114,575,374 & RP11-115D4 & Signal deleted on der(5) \\
\hline $5 q 23.1$ & chr5:1 16,078,687-116,097,905 & RP11-249M12 & Signal deleted on der(5) \\
\hline $5 q 23.2$ & chr5:123,826,090-123,997,725 & RP11-689H7 & Signal deleted on der(5) \\
\hline $5 q 23.3$ & chr5:126,087,655-126,112,178 & RP11-434D11 & Signal deleted on der(5) \\
\hline $5 q 23.3$ & chr5:130,278,846-130,432,829 & RP11-114H7 & Signal deleted on der(5) \\
\hline $5 q 31.1$ & chr5:131,789,105-131,949,164 & RP11-729C24 & Signal deleted on der(5) \\
\hline $5 q 31.1$ & chr5:135,712,100-135,888,152 & $\mathrm{RP} 11-114 \mathrm{H} 21$ & Signal deleted on der(5) \\
\hline $5 q 31.2$ & chr5:137,829,080-137,832,903 & LSI EGR1 & Signal deleted on der(5) \\
\hline $5 q 35.3$ & chr5:180,578,142-180,778,814 & D5S2907 & Signal deleted on der(5) \\
\hline $14 q 32.2$ & chr14:98,088,984-98,162,995 & RP11-76E12 & Signal on der(14); no split signal \\
\hline $14 q 32.2$ & chr14:100,070,893-100,092,077 & RP11-543C4 & Signal on der(2q); no split signal \\
\hline $14 q 32.33$ & Chr14:106,053,226- 106,518,932 & LSI IGH & Signal on der(2q); no split signal \\
\hline $15 q 15.1$ & chr15:41,796,423-41,967,115 & RP11-380D11 & Signal on der(15); no split signal \\
\hline $15 q 21.1$ & chr15:45,625,708-45,826,511 & RP11-519G16 & Signal on der(15); no split signal \\
\hline $15 q 21.1$ & chr15:46,258,718-46,459,407 & RP11-31508 & Signal on der(2), no split signal \\
\hline $15 q 21.1$ & chr15:48,509,039-48,663,778 & RP11-154J22 & Signal on der(2), no split signal \\
\hline $15 q 21.1$ & chr15:49,049,766-49,223,905 & RP11-485010 & Signal on der(2);no split signal \\
\hline $15 q 21.1$ & chr15:49,896,865-50,081,728 & RP11-353B9 & Signal on der(2); no split signal \\
\hline $15 q 21.2$ & chr15:50,385,284-50,543,688 & RP11-416K5 & Signal on der(2); no split signal \\
\hline $15 q 21.2$ & chr15:50,586,357-50,763,569 & RP11-802B2 & Signal on der(2); no split signal \\
\hline $15 q 21.3$ & $\operatorname{chr15:53,791,870-53,948,902}$ & RP11-232J12 & Signal on der(2); no split signal \\
\hline
\end{tabular}

defects would lead to progression towards AML or 5q- MDS [10].

Harrison et al., succeeded in establishing that patients with $5 \mathrm{q}$ abnormalities $(11 / 729)$ has a Hazard Ratio $=3.75$ $(1-14, p=0.01)$ in relation to patients without these abnormalities, when studying the event free survival [1]. However, it was impossible to find similar statistical results in the other survival outcomes (disease free survival and overall survival), possibly because of the small number of patients in this subgroup. Both COG protocols and United Kingdom Medical Research Council Treatment Trials AML 10 and 12 stratify these patients in the high-risk arm what makes them candidates to allogeneic bone marrow transplantation (BMT).

In another clinical study, Von Neuhoff et al., was unable to demonstrate the worst prognostic implication of aberrations in chromosome 5q (14/454) [4]. Although, it is not possible to determine if this inability was also due to a small number of patients. Patients with these types of cytogenetic abnormalities are classified in AML-BFM 2012 protocols as 
an intermediate risk group, and are not primarily directed to allogeneic BMT. Our patient was treated under the above-mentioned protocol and, because of a late achievement of complete remission; he was posteriorly stratified to high-risk group and submitted to BMT. Thus, if this uncommon breakpoint in 5q22 within complex karyotype has a prognostic impact, more studies with a wide series of AML cases should be performed to confirm our cytogenetic data.

\section{Conclusion}

Here, we described a case presenting an uncommon deletion 5q22 associated with novel abnormalities involving both chromosomes 2 , revealed by $\mathrm{MCB}$ and $\mathrm{BAC}$ probes, as defined: $46, \mathrm{XY}, \mathrm{t}(2 ; 14)(\mathrm{q} 23 ; \mathrm{q} 32.2), \mathrm{t}(2 ; 15)(\mathrm{p} 23 ; \mathrm{q} 15), \mathrm{del}(5)$ (q22-qter) in a child that showed a very poor clinical response to treatment. The knowledge of more complete descriptions of such cases harboring 5q- abnormalities, obtained through the application of molecular cytogenetic approaches, are necessary to precisely stratify the risk of this rare subgroup in childhood AML.

\section{Consent}

Written informed consent was obtained from the patient for publication of this Case report and any accompanying images. A copy of the written consent is available for review by the Editor-in-Chief of this journal.

\author{
Abbreviations \\ AML: Acute myeloid leukemia; APC: Adenomatous Polyposis coli; \\ BAC: Bacterial artificial chromosome; BMT: Bone marrow transplantation; \\ CD: Cluster differentiation; COG: Children Oncology Group; CTNNA1: Catenin \\ Alpha 1; DIAPH1: Diaphanous-Related Formin 1; EGR1: Early growth response \\ 1; FISH: Fluorescence in situ hybridization; GLRA1: Glycine Receptor Alpha 1; \\ HLA-DR: Human leukocyte antigen-D related; IGH: Immunoglobulin heavy; \\ IREM: Immune receptor expressed by myeloid cells; LSI: Locus specific; \\ MCB: Multicolor chromosome banding; MDS: Myelodisplastic Syndrome; \\ MPO: Mieloperoxidase; NPM1: Nucleophosmin 1; RPS14: Ribosomal \\ Protein S14; TdT: Terminal Deoxynucleotidyl Transferase; \\ UBE2D2: Ubiquitin-Conjugating Enzyme E2D 2.
}

\section{Competing interests}

The authors declare that they have no competing interests.

\section{Authors' contributions}

RCR and MGPL provided the data and revision of the clinical history of the child; AFF, RRCM, MAKO, TL, and MLMS conducted the cytogenetics FISH and MCB analyses. ESC conducted the immunophenotyping studies. AFF, EA, MGPL, MLMS and RCR revised the manuscript critically for important intellectual content, and the authors alone are responsible for the content and writing of the paper. All authors read and approved the final manuscript.

\section{Acknowledgments}

The authors acknowledge with gratitude Mariana Tavares de Souza and Daniela Ribeiro Ney Garcia. This work was supported by the CAPES/ PROBRAL/DAAD (project no. 419/14), Pro-Vita nonprofit organization, the International Outreach Program, the American Lebanese Syrian Associated Charities (ALSAC), St Jude Children's Research Hospital (Memphis, TN), and a Center of Excellence Grant, from the state of Tennessee.

\section{Author details}

'Bone Marrow Unit, Cytogenetics Department, National Cancer Institute, Rio de Janeiro, Brazil. ${ }^{2}$ Post-Graduation Program in Oncology, National Cancer Institute, Rio de Janeiro, Brazil. Institute for Human Genetics, Jena University, Jena, Germany. ${ }^{4}$ Clinical Medicine Postgraduate Program, College of Medicine, Federal University, Rio de Janeiro, Brazil. ${ }^{5}$ Martagão Gesteira Institute of Pediatrics and Child Development, Federal University of Rio de Janeiro, Rio de Janeiro, Brazil. ${ }^{6}$ Department of Oncology, St. Jude Children's Research Hospital, Memphis, TN, USA. ${ }^{7}$ Bone Marrow Unit, Stem Cell Department, National Cancer Institute, Rio de Janeiro, Brazil.

Received: 25 June 2015 Accepted: 28 July 2015

Published online: 07 August 2015

\section{References}

1. Harrison CJ, Hills RK, Moorman AV, Grimwade DJ, Hann I, Webb DKH, et al. Cytogenetics of childhood acute myeloid leukemia: United Kingdom medical research council treatment trials AML 10 and 12. J Clin Oncol. 2010;28(16):2674-81.

2. Manola KN. Cytogenetics of paediatric acute myeloid leukemia. Eur J Haematol. 2009;83:391-405.

3. Johnston DL, Alonzo TA, Gerbing RB, Hirsch B, Heerema NA, Ravindranath Y, et al. Outcome of Pediatric patients with with acute myeloid leukemia (AML) and $-5 / 5 q-$ abnormalities from five pediatric AML treatment protocols: a report from the Children's Oncology Group. Pediatr Blood Cancer. 2013;60:2073-78.

4. Von Neuhoff C, Reinhardt D, Sander A, Zimmermann M, Bradtke J, Betts DR, et al. Prognostic impact of specific chromosomal aberrations in a large group of pediatric patients with acute myeloid leukemia treated uniformly according to trial AML-BFM 98. J Clin Oncol. 2010;16:2682-89.

5. Reinhardt D, Creutzig U. AML-BFM 2012: der akuten myeloischen Leukämien bei kindern und Jugendlichen Gesellschaft Für Pädiatrische onkologie und hämatologie. Version Nr.3,6.28.2013, Hannover.

6. Liehr T, Heller A, Starke H, Rubtsov N, Trifonov V, Mrasek K, et al. Microdissection based high resolution multicolor banding for all 24 human chromosomes. Int J Mol Med. 2002;9:335-9.

7. Shaffer L, McGowan-Jordan J, Schmid M. ISCN 2013. An International System for Human Cytogenetic Nomenclature (2013). Basel: Karger; 2013.

8. Bram S, Rödjer S, Swolin B. Several chromosomes involved in translocations with chromosome 5 shown with fluorescence in situ hybridization in patients with malignant myeloid disorders. Cancer Genet Cytogenet. 2004;155:74-8.

9. Volkert S, Kohlman A, Schnittger S, Kern W, Haferlach T, Haferlach C. Association of the type of $5 q$ loss with complex karyotype, clonal evolution, TP53 mutation status, and prognosis in acute myeloid leukemia and myelodysplastic syndrome. Genes Chromosome Cancer. 2014;53:402-10.

10. Eisenmann KM, Dykema KJ, Matheson SF, Kent NF, de Ward AD, West RA, et al. 5q- myelodysplastic syndromes: chromosome $5 q$ genes direct a tumor-suppression network sensing actin dynamics. Oncogene. 2009;28:3429-41.

\section{Submit your next manuscript to BioMed Central and take full advantage of:}

- Convenient online submission

- Thorough peer review

- No space constraints or color figure charges

- Immediate publication on acceptance

- Inclusion in PubMed, CAS, Scopus and Google Scholar

- Research which is freely available for redistribution 